\title{
Velocity and thermal boundary layer equations for turbulent Rayleigh-Bénard convection
}

\author{
Emily S. C. Ching $\odot^{*}$ and H. S. Leung \\ Department of Physics, The Chinese University of Hong Kong, Shatin, Hong Kong \\ Lukas Zwirner and Olga Shishkina ${ }^{\dagger}$ \\ Max Planck Institute for Dynamics and Self-Organization, Am Fassberg 17, 37077 Göttingen, Germany
}

(Received 14 March 2019; revised manuscript received 26 March 2019; published 21 October 2019)

\begin{abstract}
In turbulent Rayleigh-Bénard convection, the boundary layers are nonsteady with fluctuations, the timeaveraged large-scale circulating velocity vanishes far away from the top and bottom plates, and the motion arises from buoyancy. In this paper, we derive the full set of boundary layer equations for both the temperature and velocity fields from the Boussinesq equations for a quasi-two-dimensional flow above a heated plate, taking into account all the above effects. By solving these boundary layer equations, both the time-averaged temperature and velocity boundary layer profiles are obtained.
\end{abstract}

DOI: 10.1103/PhysRevResearch.1.033037

\section{INTRODUCTION}

Thermal convection, which is ubiquitous in nature and important in many technological processes, is often studied in the setting of Rayleigh-Bénard convection (RBC) in a closed container of fluid heated from below and cooled from above [1-4]. When the temperature difference between the bottom and top plates exceeds a certain threshold, convective motion occurs. For a container with a given geometry and within the Oberbeck-Boussinesq approximation, which ignores all the dependence of material properties on temperature except for a linear dependence of density on temperature that gives rise to a buoyancy force, the dynamics of the RBC system is determined by two dimensionless parameters: the Rayleigh number $\mathrm{Ra}=\alpha g \Delta L^{3} /(\kappa \nu)$ and the Prandtl number $\operatorname{Pr}=\nu / \kappa$. Here $\alpha$ denotes the isobaric thermal expansion coefficient, $v$ the kinematic viscosity and $\kappa$ the thermal diffusivity of the fluid, $g$ the acceleration due to gravity, $\Delta$ the temperature difference between the bottom and top plates, and $L$ the height of the container.

For a sufficiently large temperature difference between the top and bottom plates, RBC becomes turbulent and velocity boundary layers (BLs) near all rigid walls and thermal BLs near the top and bottom plates develop. Boundary layers play a crucial role in turbulent transport. The thickness of the thermal $\mathrm{BL}$ is directly related to the turbulent heat transfer. One challenge is to understand the profiles of the timeaveraged velocity and temperature fields near the boundaries. This knowledge of the spatial structure of the BLs is important for the development of reliable turbulence models for

\footnotetext{
*ching@phy.cuhk.edu.hk

†Olga.Shishkina@ds.mpg.de
}

Published by the American Physical Society under the terms of the Creative Commons Attribution 4.0 International license. Further distribution of this work must maintain attribution to the author(s) and the published article's title, journal citation, and DOI. thermal convection and for many engineering applications. A related challenge is to derive theoretically the dependence of the Nusselt number $\mathrm{Nu}$, which measures the turbulent heat transfer, and the Reynolds number Re, which measures the time-averaged velocity of the large-scale circulation (LSC), on Ra and Pr.

Grossmann and Lohse developed a scaling theory [5-8], which allows the prediction of $\mathrm{Nu}$ and $\mathrm{Re}$ as functions of $\mathrm{Ra}$ and Pr when proper prefactors [9] are used. The GrossmannLohse (GL) theory assumes that in the BL dominated regimes, the velocity BL thickness is inversely proportional to $\sqrt{\mathrm{Re}}$, a result that holds in the Prandtl-Blasius (PB) BL theory $[10,11]$ for two-dimensional (2D) steady flows. The PB theory was extended by Pohlhausen [12] to study heat transfer in forced convection. The GL theory does not give results on the spatial structure of the BLs. There have been several studies on how well the Prandtl-Blasius-Pohlhausen (PBP) theory can describe the spatial structure of the BL profiles in turbulent $\mathrm{RBC}$ and systematic deviations between the time-averaged temperature and velocity profiles measured in experimental and numerical studies from the PBP predictions were found [13-18]. The deviations are generally larger for higher Ra and lower Pr. It was suggested that the PBP predictions could be good descriptions of the BL profiles in turbulent RBC if the $\mathrm{BL}$ profiles are resampled in a dynamical frame that fluctuates with the instantaneous BL thickness [19-21]. However, deviations remain even after such a dynamical rescaling procedure is applied [18].

In turbulent RBC, the BLs are nonsteady with fluctuations, the time-averaged velocity in the direction of the LSC vanishes far away from the plates, and the motion of the fluid is not forced but arises from buoyancy. The PBP theory is a BL theory for steady forced convection with the temperature being passive and the horizontal velocity along the heated plate approaching a constant far away from the plate and Stewartson's BL theory [22] for steady free convection includes the effects of buoyancy within the Oberbeck-Boussinesq approximation. However, there is no 
BL theory that successfully incorporates all the above physical effects.

In [23], a thermal BL equation that takes into account fluctuations was derived, using a space-dependent eddy thermal diffusivity $\kappa_{t}$. For high Pr, the thermal BL is nested within the much thicker viscous BL, and as a result, $\kappa_{t}$ and the time-averaged horizontal velocity can be approximated, respectively, as a cubic and a linear function of the distance from the plate. This allows us to solve the thermal BL equation and obtain the time-averaged normalized temperature BL profile as an integral with one parameter. These theoretical results for high Pr are in good agreement with the GOLDFISH [24] direct numerical simulations (DNSs) [23] and experimental data [25]. For a general $\operatorname{Pr}$ and particularly for a low Pr, one needs to know the whole profiles of $\kappa_{t}$ and the velocity stream function in order to obtain theoretical results for the time-averaged temperature BL profiles. This calls for the derivation of the BL equation for the velocity field, which is a challenging task.

In this paper, we derive the full set of BL equations for both the temperature and velocity fields from the Boussinesq equations, taking into account all the physical effects discussed above. Fluctuations are included by using space-dependent eddy thermal diffusivity $\kappa_{t}$ and eddy viscosity $v_{t}$. The thermal and velocity $\mathrm{BL}$ equations are coupled due to buoyancy, except when the horizontal pressure gradient is negligible. Solving these BL equations, we obtain the time-averaged temperature and velocity BL profiles for a general Pr in terms of two parameters that measure the size of fluctuations.

\section{THEORY}

\section{A. The BL equations including fluctuations}

We consider a quasi-2D fluid flow above a semi-infinite horizontal heated plate in RBC. The velocity field is $\mathbf{u}(x, z, t) \equiv u(x, z, t) \hat{x}+v(x, z, t) \hat{z}$, with the vertical direction $\hat{z}$ and the horizontal direction $\hat{x}$, and the temperature field is $T(x, z, t)$. The governing Boussinesq equations are

$$
\begin{gathered}
\partial_{t} \mathbf{u}+\mathbf{u} \cdot \nabla \mathbf{u}=-\nabla p / \rho+v \nabla^{2} \mathbf{u}+\alpha g\left(T-T_{h}\right) \hat{z}, \\
\partial_{t} T+\mathbf{u} \cdot \nabla T=\kappa \nabla^{2} T, \\
\nabla \cdot \mathbf{u}=0,
\end{gathered}
$$

where $p(x, z, t)$ is the hydrodynamic pressure, $\rho$ is the density, and $T_{h}$ is the temperature of the heated plate situated at $z=0$.

The flow is unsteady with fluctuations. We perform the Reynolds decomposition of the flow fields into sums of time averages $U, V, \Theta$, and $P$ and fluctuations $u^{\prime}, v^{\prime}, \theta^{\prime}$, and $p^{\prime}$,

$$
\begin{gathered}
u=U+u^{\prime}, \\
v=V+v^{\prime}, \\
T_{h}-T=\Theta+\theta^{\prime}, \\
p=P+p^{\prime}
\end{gathered}
$$

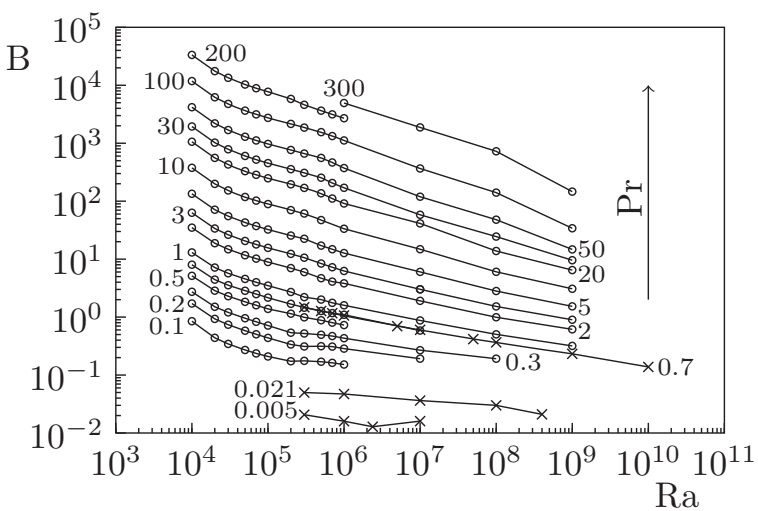

FIG. 1. Values of $B$ obtained in the DNS [8] (circles) and [26] (crosses). The data points for the same Pr, which ranges from 0.005 to 300 , are connected by a solid line.

and average (1) and (2) over time to obtain

$$
\begin{gathered}
U \partial_{x} U+V \partial_{z} U+\partial_{z}\left\langle u^{\prime} v^{\prime}\right\rangle_{t}=-\rho^{-1} \partial_{x} P+v \partial_{z}^{2} U, \\
\rho^{-1} \partial_{z} P=-\alpha g \Theta, \\
U \partial_{x} \Theta+V \partial_{z} \Theta+\partial_{z}\left\langle v^{\prime} \theta^{\prime}\right\rangle_{t}=\kappa \partial_{z}^{2} \Theta,
\end{gathered}
$$

together with the boundary conditions (BCs)

$$
\begin{gathered}
U(x, 0)=0, \quad V(x, 0)=0, \\
U(x, \infty)=0, \quad \partial_{z} U(x, \infty)=0, \\
\Theta(x, 0)=0, \quad \Theta(x, \infty)=\Delta / 2 .
\end{gathered}
$$

The BCs for $U$ in (11) and (12) reflect the fact that the timeaveraged velocity of the LSC, which develops along the plate in $\mathrm{RBC}$ at sufficiently high $\mathrm{Ra}$, achieves its maximum value at a certain distance from the plate and then vanishes far away from the plate into the central core region of the cell.

To obtain (8)-(10), we have taken the BL approximation that the partial derivatives of the flow quantities along the plate are much smaller than those away from the plate

$$
\begin{gathered}
\left|\partial_{x}^{2} U\right| \ll\left|\partial_{z}^{2} U\right|, \quad\left|\partial_{x}\left\langle u^{\prime 2}\right\rangle_{t}\right| \ll\left|\partial_{z}\left\langle u^{\prime} v^{\prime}\right\rangle_{t}\right|, \\
\left|\partial_{x}^{2} \Theta\right| \ll\left|\partial_{z}^{2} \Theta\right|, \quad\left|\partial_{x}\left\langle u^{\prime} \theta^{\prime}\right\rangle_{t}\right| \ll\left|\partial_{z}\left\langle v^{\prime} \theta^{\prime}\right\rangle_{t}\right| .
\end{gathered}
$$

We have also assumed that the convection-diffusion terms in the vertical direction are negligible compared to the buoyancy term, which is then balanced by the pressure gradient [22]. Therefore, the presence of the horizontal pressure gradient in (8) is a direct manifestation of the effect of buoyancy. This balance implies that the pressure $P$ is of the order $B \rho U^{2}$ (cf. Sec. III) with

$$
B \equiv \frac{\mathrm{Ra}}{2 \operatorname{Pr}_{\mathrm{Re}^{5 / 2}}} .
$$

Thus the horizontal pressure gradient would play a direct role in (8) when $B$ is at least of order 1. In Fig. 1 we show the values of $B$ obtained in the DNS [8,24,26,27]. It can be seen that $B$ decreases with $\operatorname{Pr}$ and $B \ll 1$ at low $\operatorname{Pr}$ and high Ra. For $B \ll 1$, the horizontal pressure gradient term is negligible 
such that (8) is decoupled from the energy equation (10). In this case, the effect of buoyancy is manifested indirectly via the $\mathrm{BC}$ of $U$, which is different from that imposed in the PBP theory or Falkner-Skan approximation [28-31] for steady forced convection where temperature is a passive scalar. Thus the problem is divided into two classes: $\mathcal{H}_{B}=0$, where $B \ll 1$ and the horizontal pressure gradient is negligible, and $\mathcal{H}_{B}=1$, where $B$ is at least of order 1 and the horizontal pressure gradient plays a direct role.

We study the correlations $\left\langle u^{\prime} v^{\prime}\right\rangle_{t}$ and $\left\langle v^{\prime} \theta^{\prime}\right\rangle_{t}$ due to fluctuations using the space-dependent eddy viscosity $v_{t}(x, z)$ and eddy thermal diffusivity $\kappa_{t}(x, z)$, which are defined by

$$
\left\langle u^{\prime} v^{\prime}\right\rangle_{t} \equiv-v_{t} \partial_{z} U, \quad\left\langle v^{\prime} \theta^{\prime}\right\rangle_{t} \equiv-\kappa_{t} \partial_{z} \Theta,
$$

and obtain the BL equations

$$
\begin{gathered}
U \partial_{x} U+\left(V-\partial_{z} v_{t}\right) \partial_{z} U=\left(v+v_{t}\right) \partial_{z}^{2} U-\mathcal{H}_{B} \rho^{-1} \partial_{x} P \\
U \partial_{x} \Theta+\left(V-\partial_{z} \kappa_{t}\right) \partial_{z} \Theta=\left(\kappa+\kappa_{t}\right) \partial_{z}^{2} \Theta
\end{gathered}
$$

together with (9). Eddy viscosity $v_{t}$ and eddy thermal diffusivity $\kappa_{t}$ are classical concepts in fluid mechanics employed to study fluid flows where the fluctuations are not negligible. To satisfy the continuity equation for the time-averaged velocity field [obtained by taking the time average of (3)], we introduce the stream function $\Psi$,

$$
U=\partial_{z} \Psi, \quad V=-\partial_{x} \Psi .
$$

\section{B. Similarity solutions}

We define the similarity variable $\xi$, the dimensionless stream function $\psi(\xi)$, the dimensionless pressure $F(\xi)$, and the dimensionless temperature $\theta(\xi)$ as

$$
\begin{gathered}
\xi \equiv \operatorname{Pr}^{a} \operatorname{Ra}^{b} \frac{z}{L}\left(\frac{x}{L}\right)^{c}, \\
\Psi \equiv \operatorname{Pr}^{a} \operatorname{Ra}^{b} \nu\left(\frac{x}{L}\right)^{d} \psi(\xi), \\
P \equiv \operatorname{Pr}^{e} \operatorname{Ra}^{f} \frac{\rho \nu^{2}}{2 L^{2}}\left(\frac{x}{L}\right)^{m} F(\xi), \\
\Theta \equiv(\Delta / 2) \theta(\xi)
\end{gathered}
$$

and look for a similarity solution of the BL equations (9), (18), and (19) in terms of $\xi$. The BCs that correspond to (11)-(13) are

$$
\begin{gathered}
\psi(0)=0, \quad \psi_{\xi}(0)=0, \\
\psi_{\xi}(\infty)=0, \quad \psi_{\xi \xi}(\infty)=0, \\
\theta(0)=0, \quad \theta(\infty)=1 .
\end{gathered}
$$

Substituting (21)-(24) into (9) and (19) and requiring the resulting equations to be independent of $x$ and $\mathrm{Ra}$, we obtain

$$
\begin{gathered}
F_{\xi}+\theta=0 \\
\left(1+\kappa_{t} / \kappa\right) \theta_{\xi \xi}+\left[\left(\kappa_{t} / \kappa\right)_{\xi}+\operatorname{Pr}(c+1) \psi\right] \theta_{\xi}=0
\end{gathered}
$$

and

$m=-c, \quad e=-(1+a), \quad f=1-b, \quad d=c+1$.
Then substituting (21)-(24) together with (30) into (18), we obtain

$$
\begin{gathered}
\left(1+\frac{v_{t}}{v}\right) \psi_{\xi \xi \xi}+\left(\frac{v_{t}}{v}\right)_{\xi} \psi_{\xi \xi}+(c+1) \psi \psi_{\xi \xi}-(2 c+1)\left(\psi_{\xi}\right)^{2} \\
=\frac{c \mathcal{H}_{B}}{2} \operatorname{Pr}^{-(5 a+1)} \operatorname{Ra}^{1-5 b}\left(\frac{x}{L}\right)^{-(5 c+2)}\left(\xi F_{\xi}-F\right) .
\end{gathered}
$$

We discuss the two classes $\mathcal{H}_{B}=1$, where the horizontal pressure gradient is acting, and $\mathcal{H}_{B}=0$, where the horizontal pressure gradient is negligible, separately below.

\section{Class $\mathcal{H}_{B}=1$}

The requirement that (31) is independent of $x, \operatorname{Pr}$, and $\mathrm{Ra}$ implies that

$$
a=-1 / 5, \quad b=1 / 5, \quad c=-2 / 5,
$$

and (31) becomes

$$
\begin{aligned}
& 5\left(1+v_{t} / v\right) \psi_{\xi \xi \xi}+5\left(v_{t} / v\right)_{\xi} \psi_{\xi \xi}+3 \psi \psi_{\xi \xi}-\left(\psi_{\xi}\right)^{2} \\
& \quad=F-\xi F_{\xi} .
\end{aligned}
$$

In this case, we have three equations (28), (29), and (33) with five unknown functions $\psi, F, \theta, v_{t}$, and $\kappa_{t}$.

\section{Class $\mathcal{H}_{B}=0$}

For $\mathcal{H}_{B}=0$, (31) reduces to

$$
\begin{aligned}
(1 & \left.+v_{t} / v\right) \psi_{\xi \xi \xi}+\left(v_{t} / v\right)_{\xi} \psi_{\xi \xi}+(c+1) \psi \psi_{\xi \xi} \\
& =(2 c+1)\left(\psi_{\xi}\right)^{2} .
\end{aligned}
$$

Equation (34) with $v_{t}=0$ is identical to the BL equation for steady plane wall jets [32]. The values of $a$ and $b$ are undetermined. We will show (see Sec. IIE2) that for (34) to have nontrivial solutions, $c$ is restricted to certain values. In this case, we have two equations (29) and (34) with four unknown functions $\psi, \theta, v_{t}$, and $\kappa_{t}$.

\section{Closure model for $v_{t}$ and $\kappa_{t}$}

To have the same number of equations and unknown functions, we need a closure model which relates $v_{t}$ and $\kappa_{t}$ to the other unknown functions. Using Prandtl's mixing length model [33], we first express $v_{t}$ and $\kappa_{t}$ in terms of the time-averaged vertical velocity gradient

$$
v_{t} / v \approx\left(l_{v}^{2} / v\right) \partial_{z} V, \quad \kappa_{t} / \kappa \approx\left(l_{v} l_{\theta} / \kappa\right) \partial_{z} V,
$$

where $l_{v}$ and $l_{\theta}$ are the mixing lengths. Near the plate, we estimate $l_{v}$ and $l_{\theta}$ to be proportional to $z$, which implies that $\left(v_{t} / \nu\right)_{\xi}$ and $\left(\kappa_{t} / \kappa\right)_{\xi}$ are proportional to $\left(z^{2} \partial_{z} V\right)_{\xi} \propto \psi$. Thus we propose the closure model

$$
\begin{aligned}
& \left(v_{t} / v\right)_{\xi} \approx k_{1} \psi, \\
& \left(\kappa_{t} / \kappa\right)_{\xi} \approx k_{2} \psi,
\end{aligned}
$$

with positive constants $k_{1}$ and $k_{2}$. This closure model extends the idea of [34] and can be written as

$$
v_{t} / \nu \approx k_{1} \int_{0}^{\xi} \psi(p) d p=k_{1} G, \quad \kappa_{t} / \kappa \approx k_{2} G,
$$


where

$$
G \equiv \int_{0}^{\xi} \psi(p) d p
$$

\section{High-Pr limit}

Equations (29) and (37) lead to

$$
\left(1+\kappa_{t} / \kappa\right) \theta_{\xi \xi}+\left[1+(c+1) \operatorname{Pr} / k_{2}\right]\left(\kappa_{t} / \kappa\right)_{\xi} \theta_{\xi}=0,
$$

which implies

$$
\theta(\xi)=\frac{\int_{0}^{\xi}\left[1+k_{2} \int_{0}^{\eta} \psi(q) d q\right]^{-C} d \eta}{\int_{0}^{\infty}\left[1+k_{2} \int_{0}^{\eta} \psi(q) d q\right]^{-C} d \eta},
$$

where

$$
C \equiv 1+\frac{(c+1) \operatorname{Pr}}{k_{2}}
$$

and we have used (27) and (38). In general, one needs to know the whole profile of $\psi(\xi)$ in order to obtain $\theta(\xi)$. An exception occurs in the limit of high Pr.

For high Pr, the thermal BL is nested inside the much thicker velocity BL. Thus, in the high-Pr limit, we can take the approximation

$$
\psi(\xi) \approx \psi_{\xi \xi}(0) \xi^{2} / 2
$$

in (41) and obtain

$$
\theta(\xi)=\frac{\int_{0}^{\xi}\left(1+\hat{A}^{3} \eta^{3}\right)^{-\left(1+1 / K_{2}\right)} d \eta}{\int_{0}^{\infty}\left(1+\hat{A}^{3} \eta^{3}\right)^{-\left(1+1 / K_{2}\right)} d \eta},
$$

where $\hat{A}^{3} \equiv \psi_{\xi \xi}(0) k_{2} / 6$. We can further take $\zeta=\theta_{\xi}(0) \xi$ to obtain the normalized temperature BL profile

$$
\theta(\zeta)=\int_{0}^{\zeta}\left(1+A^{3} \eta^{3}\right)^{-C} d \eta, \quad \theta_{\zeta}(0)=1,
$$

where

$$
A=\frac{\hat{A}}{\theta_{\xi}(0)}=\frac{\psi_{\xi \xi}(0) k_{2}}{6 \theta_{\xi}(0)}
$$

is related to $C$ by the requirement $\theta(\infty)=1$,

$$
A=\frac{\Gamma(1 / 3) \Gamma(C-1 / 3)}{3 \Gamma(C)},
$$

where $\Gamma$ is the Gamma function.

The theoretical result (45) with (47) for the time-averaged normalized temperature BL profile for high-Pr has been reported in [23]. There is one independent parameter $C$ which is a function of the size of fluctuations measured by $k_{2}$. In the steady-flow or zero-fluctuation limit, $k_{2} \rightarrow 0$ and $C \rightarrow \infty$ $(A \rightarrow 0)$, Eq. (45) becomes identical to the normalized temperature BL profile obtained by the PBP theory in the high-Pr limit. This is not surprising because in the high-Pr limit, $\theta(\zeta)$ is independent of the details of $\psi$ and relies only on the small$\xi$ expansion (43) of $\psi$, which is valid for all possible solutions of $\psi$. In the large-fluctuation limit, $k_{2} \rightarrow \infty$ and $C \rightarrow 1$ $[A \rightarrow 2 \pi /(3 \sqrt{3})]$ and the integral in (45) can be evaluated analytically [23]. Hence, in the high-Pr limit, the normalized temperature $\mathrm{BL}$ profile deviates from the prediction of the PBP theory solely because of the presence of fluctuations that are captured by $\kappa_{t}$ and measured by $k_{2}$. It has been shown [23] that the DNS results for the temperature profiles, averaged in time and over the horizontal cross sections, for $\operatorname{Pr}=4.38$ and $\operatorname{Pr}=2547.9$ with $\mathrm{Ra}=10^{7}-10^{10}$, deviate from the PBP prediction but can be well described by (45) with $C=1$ and $C=2$, respectively. This clearly demonstrates that the fluctuations in the BLs in turbulent RBC cannot be neglected.

\section{E. General Pr}

To obtain the time-averaged temperature BL profile for a general $\operatorname{Pr}$ and particularly for low $\mathrm{Pr}$, we need to know the whole profile of $\psi(\xi)$, which was not available before but can now be obtained by solving the velocity BL equation. With the full set of BL equations for both the velocity and temperature field derived, there is no need to invoke an additional assumption of a model form for $\kappa_{t}$ or $\psi$ [34], and we can obtain the time-averaged velocity BL profile as well.

\section{Class $\mathcal{H}_{B}=1$}

The two coupled BL equations are obtained from (29) and (33) together with (32) and (38). Expressed in terms of $F$ and $G$, they become

$$
\begin{aligned}
\left(G_{\xi \xi}\right)^{2}-3 G_{\xi} G_{\xi \xi \xi}= & 5\left[\left(1+k_{1} G\right) G_{\xi \xi \xi \xi}+k_{1} G_{\xi} G_{\xi \xi \xi}\right] \\
& +\xi F_{\xi}-F \\
5\left(1+k_{2} G\right) F_{\xi \xi \xi} & +\left(3 \operatorname{Pr}+5 k_{2}\right) G_{\xi} F_{\xi \xi}=0 .
\end{aligned}
$$

The BCs are

$$
\begin{aligned}
G(0) & =G_{\xi}(0)=G_{\xi \xi}(0)=0, \\
G_{\xi \xi}(\infty) & =G_{\xi \xi \xi}(\infty)=0, \\
F_{\xi}(0) & =0, \quad F_{\xi}(\infty)=-1 .
\end{aligned}
$$

The dimensionless stream function and temperature $\psi(\xi)$ and $\theta(\xi)$ are obtained from $F$ and $G$ by

$$
\psi=G_{\xi}, \quad \theta=-F_{\xi},
$$

where the parameters $k_{1}$ and $k_{2}$ measure the size of the fluctuations. Equations (48) and (49) reduce to Stewartson's BL equations for steady free convection flow [22] when $k_{1}=$ $k_{2}=0$. Thus we generalize Stewartson's BL theory to include fluctuations and show that it is not applicable for $B \ll 1$ even in the steady-flow limit.

\section{Class $\mathcal{H}_{B}=\mathbf{0}$}

Equation (34) has nontrivial solutions of $\psi$ only for certain values of $c$. We show this by extending the work of Glauert [32] to the case with fluctuations described by (36). We rewrite (34) as

$$
\left[\left(1+v_{t} / v\right) \psi_{\xi \xi}\right]_{\xi}+(c+1) \psi \psi_{\xi \xi}-(2 c+1)\left(\psi_{\xi}\right)^{2}=0,
$$

integrate (53) from $\xi$ to infinity, and use (26) to obtain

$$
\left(1+v_{t} / v\right) \psi_{\xi \xi}+(c+1) \psi \psi_{\xi}+(3 c+2) E(\xi)=0,
$$

where

$$
E(\xi) \equiv \int_{\xi}^{\infty}\left[\psi_{\eta}(\eta)\right]^{2} d \eta
$$


Note that $E(0)$ is proportional to the total kinetic energy of the BL flow, which is bounded, and thus $E(\xi)$ is finite and non-negative for all values of $\xi$. Substituting (38) into (54), multiplying by $\psi_{\xi}$, and integrating the resulting equation from $\xi$ to infinity, we obtain

$$
\begin{aligned}
& \left(1+k_{1} G\right)\left(\psi_{\xi}\right)^{2}+\left(k_{1}-2 c-2\right) \psi E(\xi) \\
& \quad+\left(k_{1}-8 c-6\right) \int_{\xi}^{\infty} \psi_{\eta}(\eta) E(\eta) d \eta=0 .
\end{aligned}
$$

At $\xi=0$, using (25), Eq. (56) is reduced to

$$
\left(k_{1}-8 c-6\right) \int_{0}^{\infty} \psi_{\eta}(\eta) E(\eta) d \eta=0 .
$$

We assume that the LSC does not reverse its direction as one moves from the plate to the bulk, that is, $U \geqslant 0$, and this implies $\psi_{\xi} \geqslant 0$ for all $\xi$. Since both $\psi_{\xi}$ and $E(\xi)$ are non-negative functions of $\xi$, for a nontrivial solution of $\psi$ to exist, the coefficient in front of the integral in (57) must be equal to zero, which leads to

$$
c=-3 / 4+k_{1} / 8 \text {. }
$$

Substituting (38) and (58) in (29) and (34), we obtain the decoupled velocity and thermal BL equations in terms of $G$ and $\theta$,

$$
\begin{gathered}
8\left(1+k_{1} G\right) G_{\xi \xi \xi \xi}+\left(2+9 k_{1}\right) G_{\xi} G_{\xi \xi \xi} \\
+2\left(2-k_{1}\right)\left(G_{\xi \xi}\right)^{2}=0, \\
8\left(1+k_{2} G\right) \theta_{\xi \xi}+\left[\operatorname{Pr}\left(2+k_{1}\right)+8 k_{2}\right] G_{\xi} \theta_{\xi}=0 .
\end{gathered}
$$

Hence (59) and (60) with the BCs given by (27) and (50) summarize the BL theory for free convection with fluctuations for $B \ll 1$. The effect of buoyancy is manifested indirectly via the BCs for $G$ or $\psi$.

\section{SCALING RELATION OF VELOCITY BL THICKNESS}

In this section we justify (18) and derive the scaling relation of velocity BL thickness with Re. Using (20)-(23) with (30), we obtain

$$
\begin{gathered}
U=\left(\operatorname{Pr}^{a} \operatorname{Ra}^{b}\right)^{2} \frac{v}{L}\left(\frac{x}{L}\right)^{2 c+1} \psi_{\xi}(\xi), \\
P=\frac{\operatorname{Ra}}{2 \operatorname{Pr}\left(\operatorname{Pr}^{a} \operatorname{Ra}^{b}\right)} \frac{\rho v^{2}}{L^{2}}\left(\frac{x}{L}\right)^{-c} F(\xi),
\end{gathered}
$$

where $c$ is given by (32) and (58) for $\mathcal{H}_{B}=1$ and $\mathcal{H}_{B}=0$, respectively. We define $\mathrm{Re}$ using the maximum time-averaged horizontal velocity

$$
U_{m} \equiv(1 / L) \int_{0}^{L} \max _{z}\{U(x, z)\} d x
$$

and obtain

$$
\operatorname{Re} \equiv \frac{U_{m} L}{v}=\frac{\psi_{\xi}\left(\xi_{m}\right)}{2(c+1)}\left(\operatorname{Pr}^{a} \operatorname{Ra}^{b}\right)^{2} \equiv C_{0}^{2}\left(\operatorname{Pr}^{a} \operatorname{Ra}^{b}\right)^{2},
$$

where $\psi_{\xi}$ attains its maximum value at $\xi=\xi_{m}$. Thus

$$
\frac{P}{\rho U^{2}}=C_{0}^{5} \frac{\mathrm{Ra}}{2{\operatorname{Pr} \mathrm{Re}^{5 / 2}}}\left(\frac{x}{L}\right)^{-(5 c+2)} \frac{F(\xi)}{\left[\psi_{\xi}(\xi)\right]^{2}}
$$

and $P \sim B \rho U^{2}$, where $B$ is defined in (16), justifying (18). Then we define the velocity BL thickness $\delta(x)$ as the distance at which $U(x, z)$ attains its maximum value, which is given by $\xi[z=\delta(x)]=\xi_{m}$. This implies that

$$
\frac{\delta(x)}{L}=\frac{C_{0}}{\sqrt{\mathrm{Re}}} \xi_{m}\left(\frac{x}{L}\right)^{-c} .
$$

Hence, in turbulent $\mathrm{RBC}, \delta(x)$ is also inversely proportional to $\sqrt{\mathrm{Re}}$, albeit with an $x$ dependence different from that of the PBP theory. Such a scaling relation can be obtained by balancing $V \partial_{z} U$ and $v \partial_{z}^{2} U$ in (18) and using the continuity equation for the time-averaged velocity field so it holds in general for all BL theories based on (8)-(10).

\section{COMPARING THEORETICAL AND DNS RESULTS AT LOW Pr}

The BL equations (48) and (49) for $\mathcal{H}_{B}=1$ and (59) and (60) for $\mathcal{H}_{B}=0$, together with the corresponding BCs, can be solved numerically for given values of $\operatorname{Pr}, k_{1}$, and $k_{2}$ to obtain the time-averaged temperature BL profile $\theta(\xi)$ and the time-averaged horizontal velocity BL profile normalized by its maximum $\psi_{\xi}(\xi) / \psi_{\xi}\left(\xi_{m}\right)$, where $\psi_{\xi}$ attains its maximum at $\xi_{m}$. We have shown that our theoretical results for the timeaveraged normalized temperature BL profiles in the high-Pr limit are in good agreement with DNS results for temperature profiles averaged in time and over horizontal cross sections for $\operatorname{Pr} \geqslant 4.38$ [23]. Good agreement can also be obtained with the temperature profiles, averaged in time and along the axis of the cylindrical cell. Here we compare our theoretical results for both the time-averaged temperature and velocity BL profiles with the DNS results at low $\operatorname{Pr}: \operatorname{Pr}=0.1$ and $\mathrm{Ra}=10^{8}$. Using the DNS data, we evaluate the temperature profile averaged in time along the axis of the cylindrical cell. This allows us to make a direct comparison with the theoretical results. To obtain the velocity profile, we determine the direction of the LSC near the hot plate and evaluate the profile of the velocity component in this fixed direction averaged over time. At $\operatorname{Pr}=0.1$ and $\mathrm{Ra}=10^{8}$, the value of

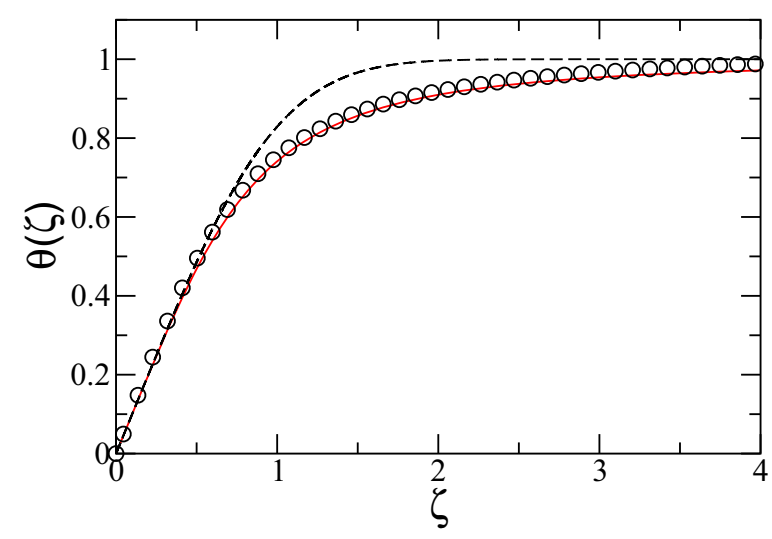

FIG. 2. Comparison of the DNS temperature profile (circles), averaged in time along the axis of a cylindrical cell, at $\operatorname{Pr}=0.1$ and $\mathrm{Ra}=10^{8}$ with the theoretical result $\theta(\zeta)$ (solid line). We show also the results from the PBP theory for $\operatorname{Pr}=0.1$ (dashed lines). Here $\zeta \propto \xi$ is chosen such that $\theta_{\zeta}(0)=1$. 


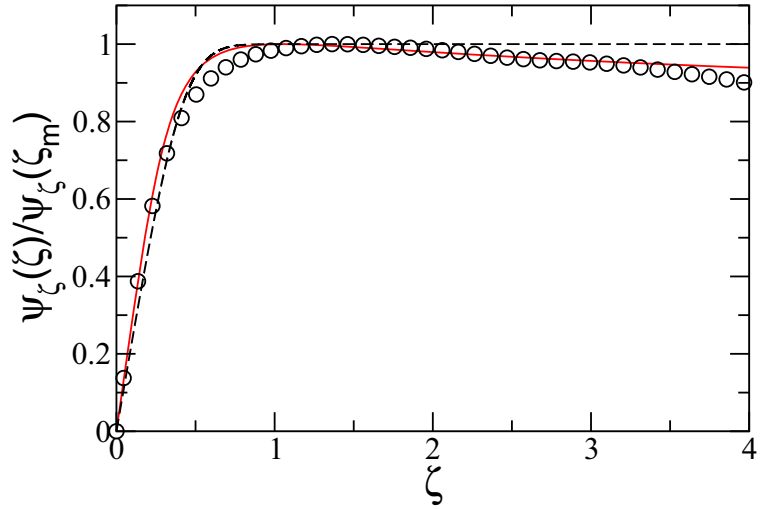

FIG. 3. Comparison (similar to Fig. 2) of the DNS profile of the velocity component in the LSC direction near the hot plate (circles), averaged in time and along the axis of the cylindrical cell and normalized by its maximum value, with the theoretical result $\psi_{\zeta}(\zeta) / \psi_{\zeta}\left(\zeta_{\mathrm{m}}\right)$ (solid line). We show also the results from the PBP theory for $\operatorname{Pr}=0.1$ (dashed lines). Here $\zeta \propto \xi$ is chosen such that $\theta_{\zeta}(0)=1$.

$B$ is found to be 0.082 , which corresponds to the case of $\mathcal{H}_{B}=0$. We solve the $\mathrm{BL}$ equations for $\mathcal{H}_{B}=0$ at $\operatorname{Pr}=0.1$ and choose the values $k_{1}$ and $k_{2}$ that give the best fits to both the DNS time-averaged temperature and velocity profiles. The best-fit values are $k_{1}=1.65$ and $k_{2}=0.138$. We show the comparisons for the time-averaged temperature and velocity BL profiles in Figs. 2 and 3, respectively.

As shown in Fig. 2, the theoretical result is in good agreement with the DNS time-averaged temperature profile. In Fig. 3 it can be seen that the theoretical result is a good approximation of the DNS time-averaged velocity profile for $0 \leqslant \zeta \leqslant 3$ and this approximation is better than existing $\mathrm{BL}$ theories. We note the inherent difficulty in making the comparison of the DNS time-averaged velocity profile with the theoretical result due to the change of the orientation of the LSC as one moves away from the hot plate found in the DNS. One might be able to obtain better agreement if this change in orientation can be corrected [35].

\section{SUMMARY}

We have derived the full set of BL equations for both the velocity and temperature fields in turbulent $\mathrm{RBC}$ from the quasi-2D Boussinesq equations. Our theory takes into account various physical effects that were not included in existing BL theories, namely, that the motion is not forced but arises from buoyancy, the BLs are nonsteady with fluctuations, and the time-averaged velocity component in the direction of the LSC vanishes as one moves into the central core region of the convection cell. We have used the commonly employed concepts of eddy viscosity $v_{t}$ and eddy thermal diffusivity $\kappa_{t}$ to study fluctuations and proposed a closure model to relate $v_{t}$ and $\kappa_{t}$ to the stream function $\psi$. The effect of buoyancy is manifested either directly by the presence of a horizontal pressure gradient term in the velocity BL equation or indirectly by the boundary condition for the horizontal velocity component far away from the plate.

This full set of BL equations enables us to obtain the time-averaged velocity and temperature BL profiles, in the form of similarity solutions, for general Pr in terms of two parameters $k_{1}$ and $k_{2}$ that measure the size of fluctuations, thus significantly extending our earlier work which gives theoretical results for the time-averaged normalized temperature profiles in the high-Pr limit [23]. We have compared our theoretical results for both the time-averaged velocity and temperature profiles against the DNS results at low Pr and demonstrated that with a suitable choice of $k_{1}$ and $k_{2}$, our theoretical results are good approximations of the DNS results at $\operatorname{Pr}=0.1$, and these approximations are more accurate than those of the existing BL theories.

We have further shown that the velocity BL thickness $\delta(x)$ is also inversely proportional to $\sqrt{\mathrm{Re}}$ in turbulent $\mathrm{RBC}$, albeit with a dependence on $x$ different from that of the PBP theory, and that Stewartson's BL theory for steady free convection is not applicable for $B \ll 1$. Moreover, the good agreement of the DNS time-averaged temperature profiles in the high-Pr limit with our theoretical results and their deviation from the $\mathrm{PBP}$ prediction is a clear demonstration that the fluctuations in BLs in turbulent RBC cannot be neglected.

With the full set of the BL equations derived, we can study how the time-averaged velocity and temperature BL profiles depend on the parameters $k_{1}, k_{2}$, and Pr. Moreover, we can study the dependence of $\mathrm{Nu}$ on the various parameters of the theory. These studies, however, are beyond the scope of the present work.

\section{ACKNOWLEDGMENTS}

O.S. and L.Z. acknowledge financial support from the Deutsche Forschungsgemeinschaft under Grants No. Sh405/4-2 (Heisenberg Fellowship) and No. Sh405/7 (Priority Programme SPP1881 "Turbulent superstructures"), respectively. The authors thank the Leibniz Supercomputing Centre for providing computing time.
[1] G. Ahlers, S. Grossmann, and D. Lohse, Heat transfer and large scale dynamics in turbulent Rayleigh-Bénard convection, Rev. Mod. Phys. 81, 503 (2009).

[2] E. Bodenschatz, W. Pesch, and G. Ahlers, Recent developments in Rayleigh-Bénard convection, Annu. Rev. Fluid Mech. 32, 709 (2000).

[3] F. Chillà and J. Schumacher, New perspectives in turbulent Rayleigh-Bénard convection, Eur. Phys. J. E 35, 58 (2012).
[4] E. S. C. Ching, Statistics and Scaling in Turbulent RayleighBénard Convection (Springer, Singapore, 2014).

[5] S. Grossmann and D. Lohse, Scaling in thermal convection: A unifying theory, J. Fluid Mech. 407, 27 (2000).

[6] S. Grossmann and D. Lohse, Thermal Convection for Large Prandtl Numbers, Phys. Rev. Lett. 86, 3316 (2001).

[7] S. Grossmann and D. Lohse, Multiple scaling in the ultimate regime of thermal convection, Phys. Fluids 23, 045108 (2011). 
[8] O. Shishkina, M. S. Emran, S. Grossmann, and D. Lohse, Scaling relations in large-Prandtl-number natural thermal convection, Phys. Rev. Fluids 2, 103502 (2017).

[9] R. J. A. M. Stevens, E. P. van der Poel, S. Grossmann, and D. Lohse, The unifying theory of scaling in thermal convection: The updated prefactors, J. Fluid Mech. 730, 295 (2013).

[10] L. Prandtl, Über Flüssigkeitsbewegung bei Sehr Kleiner Reibung, in Verhandlungen des III. Internationalen Mathematiker Kongresses, Heidelberg, 1904 (Teubner, Leipzig, 1905), pp. 484-491.

[11] L. D. Landau and E. M. Lifshitz, Fluid Mechanics, 2nd ed., Course of Theoretical Physics Vol. 6 (Butterworth Heinemann, Oxford, 1987).

[12] E. Pohlhausen, Der wärmeaustausch zwischen festen körpern und flüssigkeiten mit kleiner reibung und kleiner wärmeleitung, Z. Angew. Math. Mech. 1, 115 (1921).

[13] R. du Puits, C. Resagk, A. Tilgner, F. H. Busse, and A. Thess, Structure of thermal boundary layers in turbulent RayleighBénard convection, J. Fluid Mech. 572, 231 (2007).

[14] O. Shishkina and A. Thess, Mean temperature profiles in turbulent Rayleigh-Bénard convection of water, J. Fluid Mech. 633, 449 (2009).

[15] N. Shi, M. S. Emran, and J Schumacher, Boundary layer structure in turbulent Rayleigh-Bénard convection, J. Fluid Mech. 706, 5 (2012).

[16] J. D. Scheel, E. Kim, and K. R. White, Thermal and viscous boundary layers in turbulent Rayleigh-Bénard convection, J. Fluid Mech. 711, 281 (2012).

[17] M. Kaczorowski, O. Shishkina, A. Shishkin, C. Wagner, and K.-Q. Xia, in Direct and Large-Eddy Simulation VIII, edited by H. Kuerten, B. Geurts, V. Armenio, and J. Fröhlich (Springer, Dordrecht, 2011), pp. 383-388.

[18] R. J. A. M. Stevens, Q. Zhou, S. Grossmann, R. Verzicco, K.-Q. $\mathrm{Xia}$, and D. Lohse, Thermal boundary layer profiles in turbulent Rayleigh-Bénard convection in a cylindrical sample, Phys. Rev. E 85, 027301 (2012).

[19] Q. Zhou and K.-Q. Xia, Measured Instantaneous Viscous Boundary Layer in Turbulent Rayleigh-Bénard Convection, Phys. Rev. Lett. 104, 104301 (2010).

[20] Q. Zhou, R. J. A. M. Stevens, K. Sugiyama, S. Grossmann, D. Lohse, and K.-Q. Xia, Prandtl-Blasius temperature and velocity boundary-layer profiles in turbulent Rayleigh-Bénard convection, J. Fluid Mech. 664, 297 (2010).

[21] Q. Zhou, K. Sugiyama, R. J. A. M. Stevens, S. Grossmann, D. Lohse, and K.-Q. Xia, Horizontal structures of velocity and temperature boundary-layers in two-dimensional numerical turbulent Rayleigh-Bénard convection, Phys. Fluids 23, 125104 (2011).

[22] K. Stewartson, On the free convection from a horizontal plate, Z. Angew. Math. Phys. 9, 276 (1958).

[23] O. Shishkina, S. Horn, S. Wagner, and E. S. C. Ching, Thermal Boundary Layer Equation for Turbulent Rayleigh-Bénard Convection, Phys. Rev. Lett. 114, 114302 (2015).

[24] G. L. Kooij, M. A. Botchev, E. M.A. Frederix, B. J. Geurts, S. Horn, D. Lohse, E. P. van der Poel, O. Shishkina, R. J. A. M. Stevens, and R. Verzicco, Comparison of computational codes for direct numerical simulations of turbulent Rayleigh-Bénard convection, Comput. Fluids 166, 1 (2018).

[25] Y. Wang, X. He, and P. Tong, Boundary layer fluctuations and their effects on mean and variance temperature profiles in turbulent Rayleigh-Bénard convection, Phys. Rev. Fluids 1, 082301(R) (2016).

[26] J. D. Scheel and J. Schumacher, Predicting transition ranges to fully turbulent viscous boundary layers in low Prandtl number convection flows, Phys. Rev. Fluids 2, 123501 (2017).

[27] J. D. Scheel, M. S. Emran, and J. Schumacher, Resolving the fine-scale structure in turbulent Rayleigh-Bénard convection, New J. Phys. 15, 113063 (2013).

[28] V. M. Falkner and S. W. Skan, Some approximate solutions of the boundary layer equations, Philos. Mag. 12, 865 (1931).

[29] O. Shishkina, S. Horn, and S. Wagner, Falkner-Skan boundary layer approximation in Rayleigh-Bénard convection, J. Fluid Mech. 730, 442 (2013).

[30] O. Shishkina, S. Wagner, and S. Horn, Influence of the angle between the wind and the isothermal surfaces on the boundary layer structures in turbulent thermal convection, Phys. Rev. E 89, 033014 (2014).

[31] M. Ovsyannikov, D. Krasnov, M. S. Emran, and J. Schumacher, Combined effects of prescribed pressure gradient and buoyancy in boundary layer of turbulent Rayleigh-Bénard convection, Eur. J. Mech. B 57, 64 (2016).

[32] M. Glauert, The wall jet, J. Fluid Mech. 1, 625 (1956).

[33] L. Prandtl, Bericht über untersuchungen zur ausgebildeten turbulenz, Z. Angew. Math. Mech. 5, 136 (1925).

[34] O. Shishkina, S. Horn, M. S. Emran, and E. S. C. Ching, Mean temperature profiles in turbulent thermal convection, Phys. Rev. Fluids 2, 113502 (2017).

[35] J. Schumacher, V. Bandaru, A. Pandey, and J. D. Scheel, Transitional boundary layers in low-Prandtl-number convection, Phys. Rev. Fluids 1, 084402 (2016). 